\title{
Observation of injection locking in an optomechanical rf oscillator
}

\author{
Mani Hossein-Zadeh ${ }^{\mathrm{a}, \mathrm{b})}$ and Kerry J. Vahala \\ Department of Applied Physics, California Institute of Technology, Pasadena, California 91125, USA
}

(Received 2 July 2008; accepted 24 October 2008; published online 14 November 2008)

\begin{abstract}
Injection locking of a radiation-pressure optomechanical oscillator is demonstrated through external modulation of the optical pump power near the optomechanical oscillation frequency. It is shown that the frequency and phase of a microtoroidal optomechanical oscillator can be locked to those of an electronic oscillator (or any other signal) that can modulate the optical input power and whose frequency is within the lock range. (c) 2008 American Institute of Physics.
\end{abstract}

[DOI: $10.1063 / 1.3028024$ ]

Injection locking is a well-known effect in both selfsustained electronic and photonic oscillators (lasers). When a periodic signal with a frequency close to the oscillation frequency and large enough amplitude is injected into a selfsustained oscillator, the phase and frequency of the oscillator can be locked to that of the injected signal. ${ }^{1}$ Here this phenomenon is investigated in an optomechanical oscillator $(\mathrm{OMO}){ }^{2-4}$ OMOs are driven by radiation pressure and rely upon "dynamic back action" created when a continuouswave (cw) optical pump is blue-detuned relative to a resonance of an optical resonator that also features high- $Q$ mechanical modes. ${ }^{5}$ The OMOs of this study are based on high- $Q$ silica microtoroids. ${ }^{2-4}$ The high optical $Q$ factor and the unique geometry of toroid resonator (that enables efficient coupling between optical and mechanical degrees of freedom through radiation pressure) lead to self-sustained $\mathrm{rf}$ mechanical oscillations of the microtoroid structure ${ }^{2-4}$ (even to gigahertz frequencies ${ }^{6}$ ). Characterization of the phase noise and oscillation frequency have confirmed that the microtoroid OMO is potentially important in certain rf-photonic systems. ${ }^{7,8}$ We demonstrate that partial amplitude modulation of the optical input power can lock both frequency and phase of the optomechanical oscillation to that of the external oscillator (used to modulate the input optical power). Preliminary experimental results confirm that injection locking in an OMO exhibits the characteristics of injection locking in electronic oscillators.

Figure 1 shows the experimental arrangement used to study injection locking in the microtoroid OMO. The optical power from a tunable laser passes through a Mach-Zehnder (MZ) modulator and is coupled into and out of the microtoroid optical resonator using a fiber-taper coupler. The amplitude of the optical input power $\left(P_{\text {in }}\right)$ is partially modulated by a single tone rf signal from a tunable rf synthesizer applied on the MZ modulator. The modulation index is thereby used to control the strength of the weaker injection-locking signal (modulated fraction of $P_{\text {in }}$ ) in comparison to the pump (cw fraction of $P_{\text {in }}$ ). The spectrum of optical power that is coupled out of the microtoroid is then analyzed in a rf spectrum analyzer. Also, the phase of the output signal is compared to that of the electronic input signal (injected signal) using a lock-in amplifier. When the amplitude of the injected signal is large enough and its frequency $\left(f_{\text {inj }}\right)$ is close to

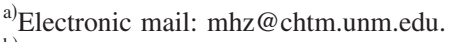

${ }^{b)}$ Present address: Center for High Technology Materials, University of New Mexico.
}

optomechanical oscillation frequency $\left(f_{\text {OMO }}\right)$, we observe injection locking of the OMO. Figure 2(a) shows the rf spectrum of the optical output power in the absence (gray trace) and presence (black trace) of modulation (injection). The microtoroid OMO chosen for this experiment had a mechanical frequency of about $84.1 \mathrm{MHz}$. In this case the frequency of the injected signal is $500 \mathrm{~Hz}$ smaller than the optomechanical oscillation frequency. The dashed trace is the rf spectrum of the optical input power when the external modulation is ON. In all experiments the laser power and amplitude modulation depth are chosen such that the cw optical input power is larger than the threshold power for optomechanical oscillation $^{3}\left(P_{\text {th }}\right)$ but small enough to keep the oscillation in the linear regime (where the second harmonic is at least 15 $\mathrm{dB}$ smaller than the fundamental frequency ${ }^{7}$ ). It is apparent that the presence of the modulation pulls $f_{\text {OMO }}$ toward $f_{\text {inj }}$ and locks the two signals. As shown in Fig. 2(b) injection locking can be used to tune the optomechanical oscillation frequency simply by tuning $f_{\text {inj. }}$. At a given ratio between modulation amplitude of the input signal and amplitude of the optomechanical oscillation imposed on the optical output $\left(A_{\text {inj }} / A_{\text {ОMO }}\right)$, locking occurs within a limited bandwidth around $f_{\text {OMO }}$ (known as lock range). Similar to an electronic oscillator, for tuning slightly above and below the edge of lock range, the oscillator is quasilocked ${ }^{9}$ and the rf spectrum consists of a series of closely spaced decaying beat frequencies in the vicinity of $f_{\text {OMO }}$ [Fig. 2(c)]. Based on the theoretical prediction using the general theory of injection locking for self-sustained oscillators, ${ }^{9}$ the lock range can be written as

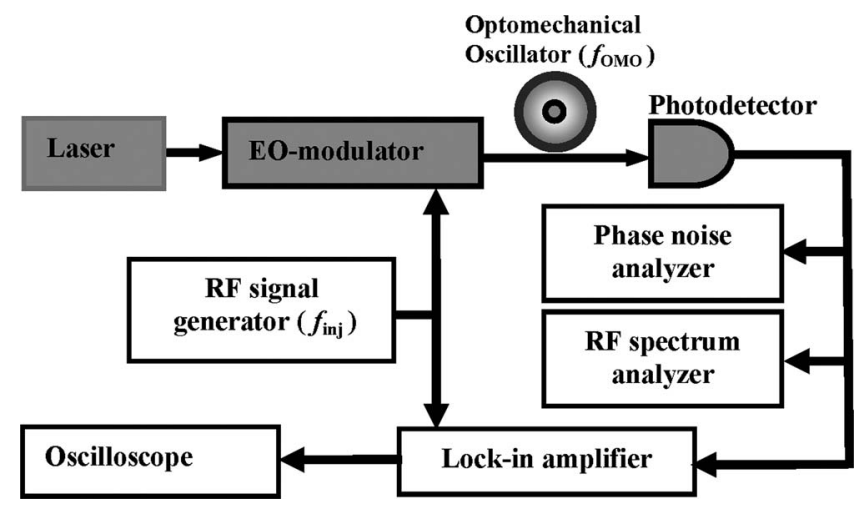

FIG. 1. Schematic diagram of the experimental arrangement used to study injection locking in a microtoroid OMO. 


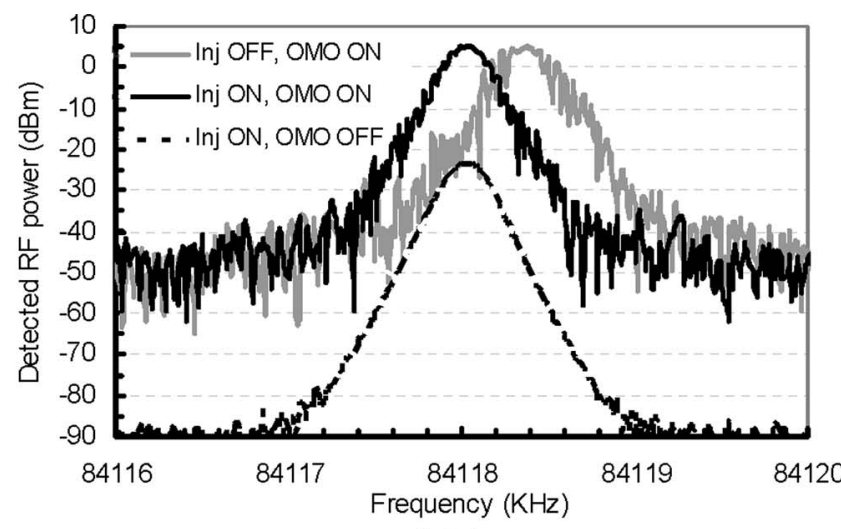

(a)

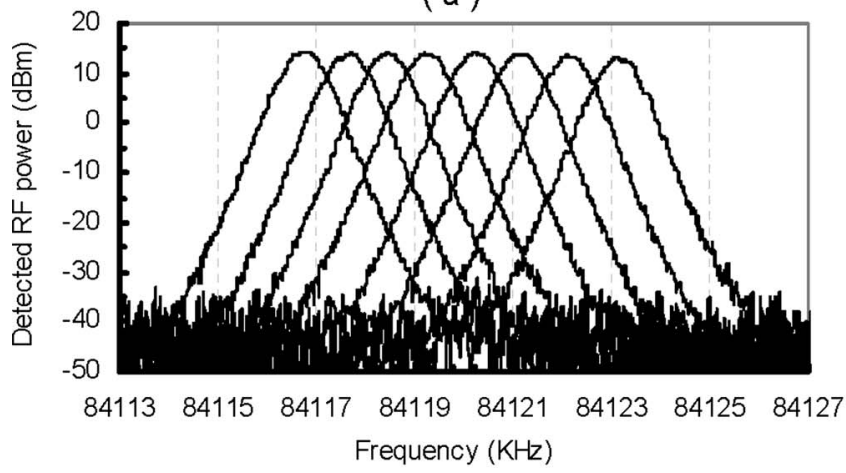

(b)

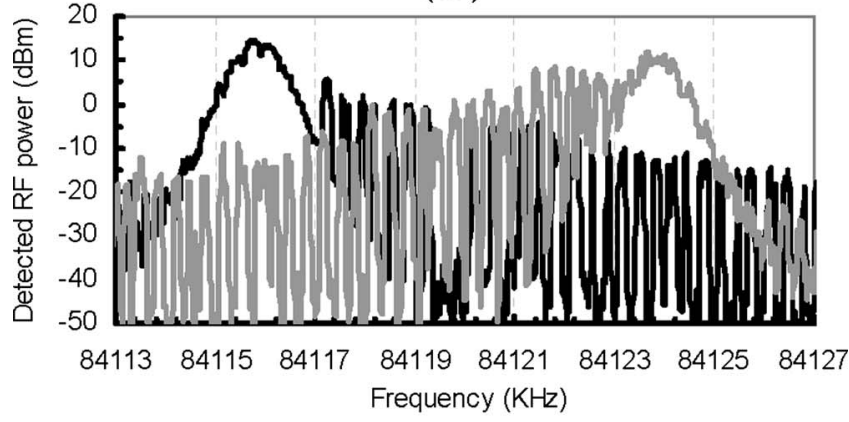

(c)

FIG. 2. (a) rf spectrum of the optical output power in the presence (black) and absence (gray) of the injection signal. The dashed trace is the spectrum of the optical input power. (b) rf spectrum of the optomechanical oscillation tuned by injection signal. (c) rf spectrum of the detected optical power while the frequency of the external modulation (injection signal) is tuned slightly above and below the lock range (quasilocked regime).

$$
\Delta f_{\text {lock }}=\Delta f_{\text {mech }} \frac{A_{\text {inj }}}{A_{\mathrm{OMO}}}\left[1-\left(\frac{A_{\text {inj }}}{A_{\mathrm{OMO}}}\right)^{2}\right]^{-1 / 2}
$$

where $\Delta f_{\text {mech }}$ is the intrinsic linewidth of the passive mechanical resonator (tank). Note that beyond the lock range the phase difference between optomechanical oscillation and the injected signal becomes random and the two signals are unlocked.

Figure 3 shows the measured lock range as a function of $A_{\text {inj }} / A_{\text {OMO }}$ for two different OMOs: one with $f_{\text {OMO }}$ $=13.8 \mathrm{MHz}$ and $\Delta f_{\text {mech }}=55 \mathrm{kHz}$ and the other with $f_{\mathrm{OMO}}$ $=84.1 \mathrm{MHz}$ and $\Delta f_{\text {mech }}=15 \mathrm{kHz}$. The solid lines are the theoretical prediction based on Eq. (1). Figure 4(a) shows the measured phase difference between the injection signal and OMO (measured using the lock-in amplifier), plotted against frequency detuning $\left(f_{\text {OMO }}-f_{\text {inj }}\right)$ within a $3.4 \mathrm{kHz}$ bandwidth around $f_{\mathrm{OMO}}$. In this measurement, $f_{\mathrm{OMO}}=13.8 \mathrm{MHz}$, $\Delta f_{\text {mech }}=55 \mathrm{kHz}$, and $A_{\text {inj }} / A_{\mathrm{OMO}}=0.36$. Figure 4(b) shows

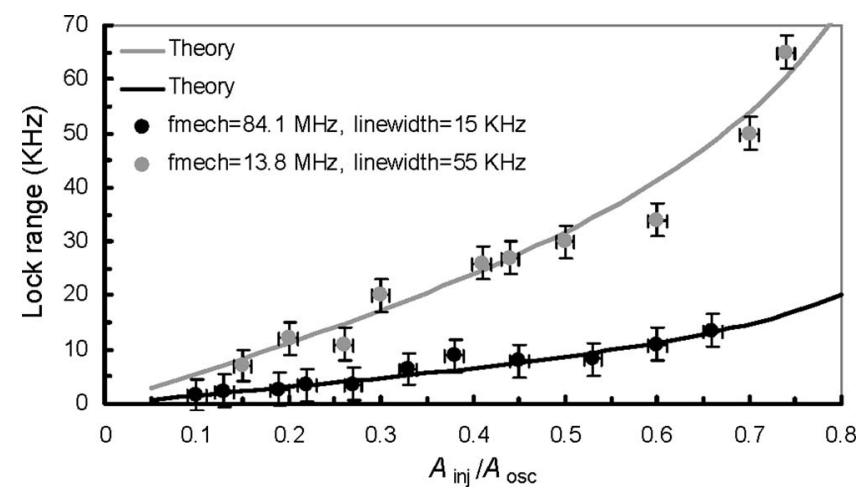

FIG. 3. Measured lock range plotted against the ratio between the modulation amplitude and optomechanical oscillation amplitude $\left(A_{\text {inj }} / A_{\text {OMO }}\right)$ for two different OMOs. The solid lines are the theoretical estimations based on Eq. (1).

the temporal behavior of the phase difference at $f_{\mathrm{OMO}}=f_{\text {inj }}$ in the presence $(\mathrm{ON})$ and absence $(\mathrm{OFF})$ of the injection signal on the MZ modulator. As evident from the trace, the phase difference remains close to zero when injection is $\mathrm{ON}$ and becomes random when injection is OFF. As a result, for $f_{\text {inj }}$ within lock range, the phase difference between the injected signal and the oscillation can be tuned by adjusting the frequency detuning.

The phase noise of the optomechanical oscillation under injection was also investigated, and as expected ${ }^{9}$ the presence of the locking signal improves the optomechanical oscillation phase noise. Figure 5 is the measured phase noise spectral density of the detected optical power for two frequency ranges (a) $0.01-10 \mathrm{kHz}$ and (b) $10-1000 \mathrm{kHz}$. The

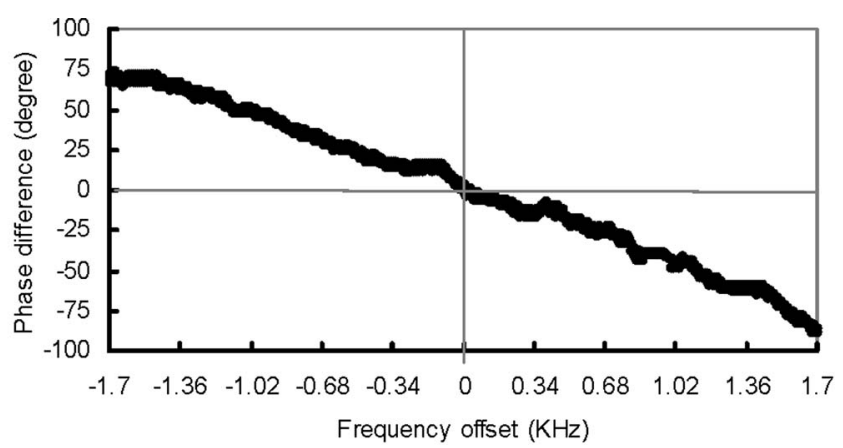

(a)

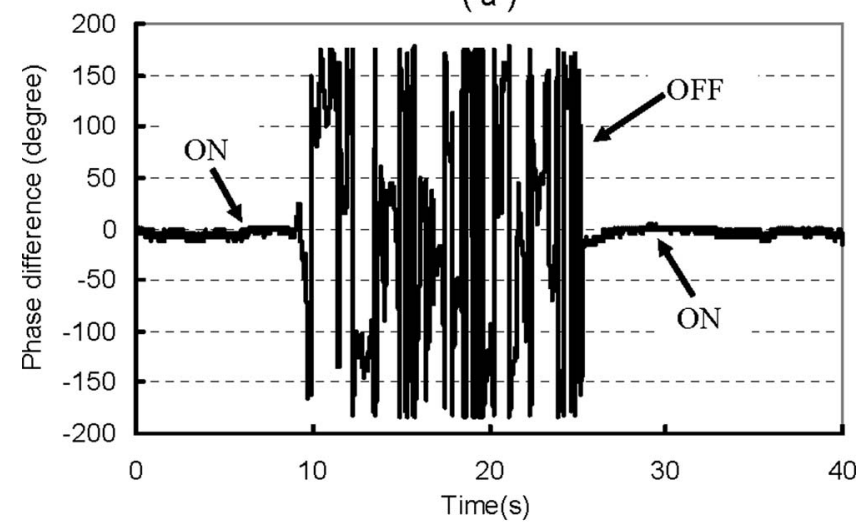

(b)

FIG. 4. (a) Measured phase difference between the injection signal and OMO in the vicinity of $f_{\mathrm{OMO}}\left(f_{\mathrm{OMO}}=13.8 \mathrm{MHz}, \Delta f_{\text {mech }}=55 \mathrm{kHz}\right.$, and $A_{\mathrm{inj}} / A_{\mathrm{OMO}}=0.36$ ). (b) Temporal behavior of the phase difference in the presence $(\mathrm{ON})$ and absence of external modulation $(\mathrm{OFF})$ when $f_{\mathrm{OMO}}=f_{\text {inj }}$. Since $f_{\mathrm{OMO}}=f_{\text {inj, }}$, the phase difference is locked to zero. 


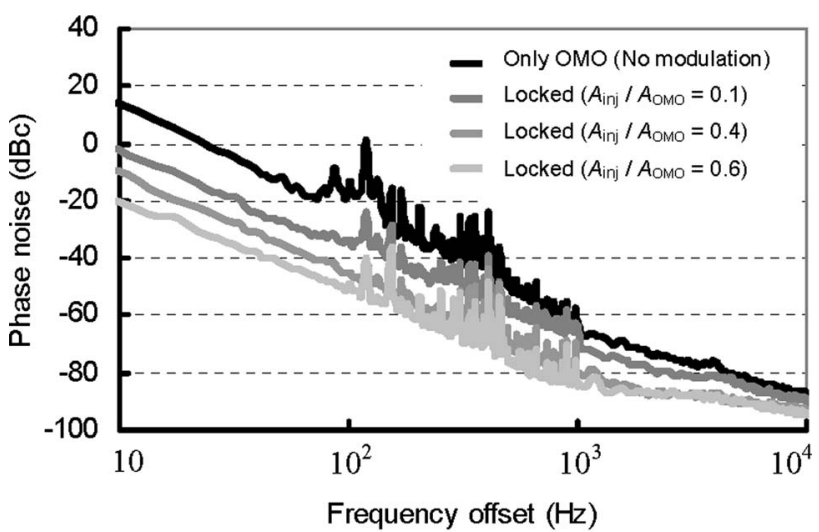

(a)

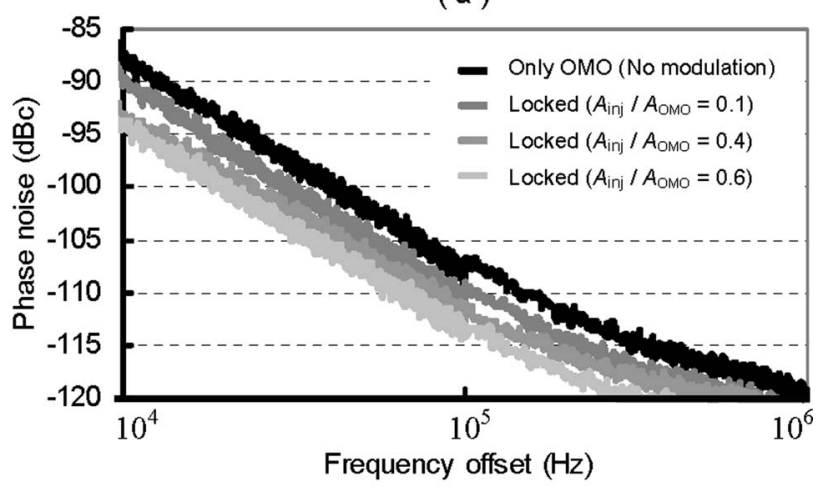

(b)

FIG. 5. Measured phase noise spectral density of the detected optical power for two frequency offset ranges (a) $0.01-10 \mathrm{kHz}$ and (b) $10-1000 \mathrm{kHz}$. The phase noise is measured for the free running OMO and also the OMO injection locked with $A_{\text {inj }} / A_{\mathrm{OMO}}=0.1,0.4$, and 0.6.

phase noise is measured for the free-running OMO and injection-locked OMO with $A_{\text {inj }} / A_{\mathrm{OMO}}=0.1,0.4$ and 0.6 (In this experiment $f_{\mathrm{OMO}}=84.1 \mathrm{MHz}$ and $\Delta f_{\text {mech }}=15 \mathrm{kHz}$ ). As evident from the noise spectrum, the phase noise improvement is especially noticeable in the low frequency range $[<10 \mathrm{kHz}$, Fig. 5(a)] because frequency locking eliminates the frequency jitter caused by slowly varying noise mechanisms. These measurements also verify that the magnitude of the phase noise improvement scales as $A_{\mathrm{inj}} / A_{\mathrm{OMO}}$.

We have also verified that, as is also true with electronic oscillators, the OMO can be injection locked to a signal with a frequency close to harmonic or subharmonic frequencies of the fundamental oscillation frequency $\left(f_{\text {OMO }}\right)$. Figure 6(a) shows the rf spectrum of the free running OMO (black trace) as well as harmonically and subharmonically locked oscillator (gray traces). In this study $f_{\mathrm{OMO}}=36.913 \mathrm{MHz}$. Figures 6(b) and 6(c) show the rf spectrum of the modulating (injected) signals for the two locked cases shown in part (a). The black vertical lines indicate the location of the second harmonic $\left(2 f_{\mathrm{OMO}}\right)$ and subharmonic $\left(f_{\mathrm{OMO}} / 2\right)$ of the main oscillation frequency. As evident from the graphs, injecting the OMO at these frequencies can also lock the frequency of the OMO to the modulating signal. The injected signals are intentionally detuned from the exact harmonic and subharmonic so that the frequency locking is clearly observable in the vicinity of $f_{\text {OMO }}$.

In conclusion we have shown that injection locking technique can be used to lock the phase and frequency of an OMO to amplitude modulation of an input optical wave. This technique, widely used in electronic and photonic systems, may open interesting frontiers in engineering of optom-

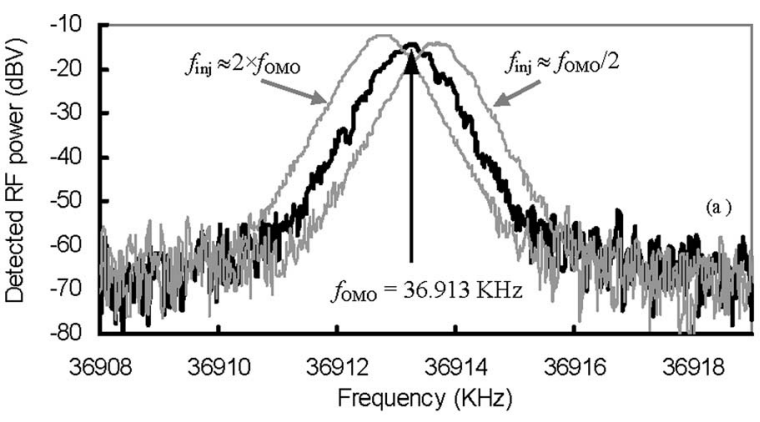

(a)

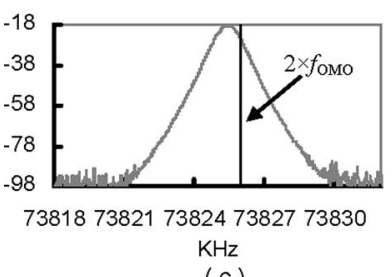

(c)

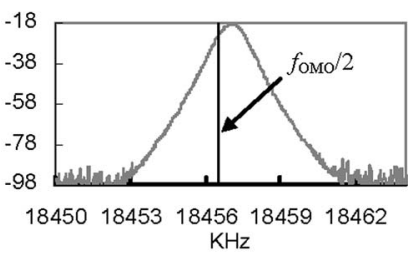

(b)
FIG. 6. (a) Measured rf spectrum of the free running OMO (black trace) and the OMO locked to subharmonic and harmonic injected signals (gray traces). (b) and (c) are the spectrums of the harmonic and subharmonic injected signals, respectively.

echanical rf oscillators and their applications. As an example, recently a photonic rf-receiver architecture based on the OMO has been demonstrated ${ }^{8}$ that can benefit from this phenomenon. In this design the locking between the optomechanical local oscillator and the received signal may relax the frequency stability requirement for the oscillator within the lock range. Injection locking of an OMO to an electronic rf oscillator enables combination of OMO with electronic devices and may have interesting applications in certain rfphotonics and micro-optoelectro-mechanical systems. Alternatively the nonelectronic nature of OMO makes them immune to electromagnetic interference and good candidates for all-optical systems. In this context injection locking can be used to lock two OMOs and improve the frequency stability and noise performance of the "slave" oscillator similar to what is done in the optical domain with lasers (Improving the performance of a noisy high power laser with a narrow linewidth low power laser). Lastly injection locking is a useful feature in metrological applications where it can be used to synchronize multiple OMOs as well as controlling optomechanical oscillations with atomic accuracy (by locking to atomic clocks).

This work was supported by Defense Advanced Research Projects Agency (DARPA), Caltech Lee Center for Advanced Networking, and Caltech Center for Physics of Information.

${ }^{1}$ R. Adler, Proc. IEEE 61, 1380 (1973).

${ }^{2}$ H. Rokhsari, T. J. Kippenberg, T. Carmon, and K. J. Vahala, Opt. Express 13, 5293 (2005).

${ }^{3}$ T. J. Kippenberg, H. Rokhsari, T. Carmon, A. Scherer, and K. J. Vahala, Phys. Rev. Lett. 95, 033901 (2005).

${ }^{4}$ T. Carmon, H. Rokhsari, L. Yang, T. J. Kippenberg, and K. J. Vahala, Phys. Rev. Lett. 94, 223902 (2005).

${ }^{5}$ T. J. Kippenberg and K. J. Vahala, Opt. Express 15, 17172 (2007).

${ }^{6}$ T. Carmon and K. J. Vahala, Phys. Rev. Lett. 98, 123901 (2007).

${ }^{7}$ M. Hossein-Zadeh, H. Rokhsari, A. Hajimiri and K. J. Vahala, Phys. Rev. A 74, 0238132006 .

${ }^{8}$ M. Hossein-Zadeh and K. J. Vahala, IEEE Photonics Technol. Lett. 20, 234 (2008).

${ }^{9}$ B. Razavi, IEEE J. Solid-State Circuits 39, 1415 (2004). 